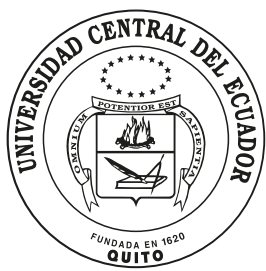

ISSN-i 1390-7042 | ISSN-e 2602-8484

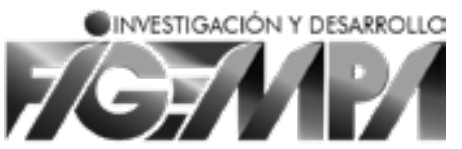

\title{
CALIDAD FISICOQUíMICA DEL AGUA DE LA LAGUNA COLTA. CHIMBORAZO, ECUADOR
}

\section{Physicochemical quality of lagoon Colta water. Chimborazo, Ecuador}

\author{
Sandra Escobar \\ Escuela Superior Politécnica del Chimborazo (Ecuador) \\ Universidad de los Andes (Venezuela) \\ kasandraea@gmail.com \\ ORCID: https://orcid.org/0000-0002-3347-0282
}

Ana Albuja

Escuela Superior Politécnica del Chimborazo (Ecuador)

Universidad de los Andes (Venezuela)

ana.albuja@espoch.edu.ec

ORCID: https://orcid.org/0000-0002-8774-279X
Felix Andueza

Universidad Central del Ecuador (Ecuador)

Universidad de los Andes (Venezuela)

fdandueza@uce.edu.ec

ORCID: https://orcid.org/0000-0002-9046-8883

\section{Recibido: mayo de 2021 \\ Aprobado: julio de 2021 ARTÍCULO ORIGINAL}

DoI: 10.29166/revfig.v11i1.3135
PALABRAS ClaVe Laguna

Colta, lagunas de alta montaña, calidad fisicoquímica.

KEYWORDs Colta lagoon, high mountain lagoons, physicochemical quality

\section{RESUMEN}

Las lagunas de alta montaña son ecosistemas muy frágiles sujetos a variaciones en las características fisicoquímicas que influyen en sus condiciones biológicas y ecológicas. En Ecuador existen una gran diversidad de lagunas de alta montaña que se han estado investigando en los últimos años. En este sentido, se realizó el presente trabajo para conocer la dinámica estacional de los factores fisicoquímicos en la laguna Colta, situada en la provincia de Chimborazo, Ecuador a una altura de $3312 \mathrm{~m} \mathrm{~s}$. n. m. Se realizaron mediciones de los parámetros fisicoquímicos conductividad eléctrica, $\mathrm{pH}$, sólidos totales disueltos, temperatura y transparencia en diferentes partes de la alguna por un periodo de dos años utilizando un equipo multiparámetro y el disco Secchi. Los resultados obtenidos indican un $\mathrm{pH}$ alcalino con valores entre 8,36 y 9,20 y un valor promedio de 8,87 , una temperatura entre 16,4 y $18,9^{\circ} \mathrm{C}$ con un valor promedio de $17,91^{\circ} \mathrm{C}$, conductividad eléctrica elevada con un rango de valores a lo largo de la laguna desde $903 \mathrm{a} 1253 \mu \mathrm{s} / \mathrm{cm}$ y un valor promedio de $1085 \mu \mathrm{s} / \mathrm{cm}$, alta cantidad de sólidos totales disueltos que variaron entre 537 a $832 \mathrm{mg} / \mathrm{L}$ y un valor promedio de $680 \mathrm{mg} / \mathrm{L}$ y un índice de transparencia Secchi indicativo de un lago eutrófico con valores entre 0,76 y 3,5 metros, con un valor promedio de 1,54 metros. Los resultados obtenidos indican un ecosistema lacustre eutrófico con parámetros fisicoquímicos estables en toda la laguna a lo largo del tiempo.

\section{ABSTRACT}

High mountain lagoons are very fragile ecosystems subject to variations in the physicochemical characteristics that influence their biological and ecological conditions. In Ecuador there is a great diversity of high mountain lagoons that have been investigated in recent years. In this sense, the present work was carried out to know the seasonal dynamics of the physicochemical factors in the Colta lagoon, located in the province of Chimborazo, Ecuador at an altitude of 3312 meters above sea level. Measurements of the physicochemical parameters, electrical conductivity, $\mathrm{pH}$, total dissolved solids, temperature and transparency were carried out in different parts of lagoon for a period of two years using a multiparameter equipment and the Seecchi disk. The results obtained indicate an alkaline $\mathrm{pH}$ with values between 8.36 and 9.20 and an average value of 8.87 , a temperate temperature between 16.4 and $18.9^{\circ} \mathrm{C}$ with an average value of $17.91{ }^{\circ} \mathrm{C}$, Elevated electrical conductivity with a range of 
values throughout the lagoon from 903 to $1253 \mu \mathrm{s} / \mathrm{cm}$ and an average value of $1085 \mu \mathrm{s} / \mathrm{cm}$, high amount of total dissolved solids that varied between 537 to $832 \mathrm{mg} / \mathrm{L}$ and an average value of $680 \mathrm{mg} / \mathrm{L}$ and a Seecchi transparency index indicative of a eutrophic lake with values between 0.76 and 3.5 meters, with an average value of 1.54 meters. The results obtained indicate a eutrophic lake ecosystem with stable physicochemical parameters throughout the lagoon over time.

\section{INTRODUCCIÓN}

Los lagos y lagunas como reservorios de agua dulce son ecosistemas muy importantes para el equilibrio ecológico y el reciclado de los elementos químicos en el planeta. Son excelentes sensores o detectores tempranos de cambios climáticos o ambientales, proporcionan información sobre las tendencias del clima a escala regional o mundial, el transporte de contaminantes atmosféricos a larga distancia, o la producción de lluvias ácidas, lo que les confiere la categoría de «ecosistemas estrella» (Granados y Toro, 2000).

Dentro de los ecosistemas lacustres encontramos las denominadas lagunas de alta montaña, ecosistemas acuáticos que se encuentran por encima de los $2200 \mathrm{~m} \mathrm{~s}$. n. m., las cuales constituyen una red de amortiguación de caudales y sedimentos provenientes de las cuencas altas y del deshielo de glaciares, constituyendo una importante reserva hídrica (Dar et al., 2020; Yarzabal et al., 2021).

Las lagunas de alta montaña son uno de los recursos más vulnerables a la contaminación y a los cambios fisicoquímicos. En particular, los lagos altoandinos tropicales son ecosistemas acuáticos con características especiales, de los cuales existen pocos estudios (Rascón et al., 2021). Principalmente, se localizan en Colombia, Ecuador y norte de Perú, entre los 2000 y $4000 \mathrm{~m}$ s. n. m. La dinámica de estos lagos tiene grandes diferencias con los lagos localizados en las zonas bajas tropicales debido a la altitud y a las condiciones climáticas dominantes (Gunkel 2003; López-Martínez et al. 2017).

En Ecuador existe una gran variedad de lagunas, tanto de alta montaña, como lacustres, cratéricas y humedales, algunas de ellas de origen volcánico sobre las cuales se han realizado algunos estudios geológicos, hidrológicos, limnológicos y químicos (Gunkel, 2003; Torres, 2016; Gómez, 2018; Tito-Ontaneda, 2018; Guaminga, 2019; Rodríguez, 2019; González et al., 2020).

En este contexto, se presenta el presente estudio en donde se investigó desde el punto de vista de la calidad fisicoquímica de sus aguas, la laguna Colta, situada en la provincia de Chimborazo a $3312 \mathrm{~m} \mathrm{s.} \mathrm{n.} \mathrm{m.}$

\section{MATERIALES Y MÉTODOS}

\section{MATERIALES}

\section{Sitio de muestreo}

El trabajo se realizó en la laguna Colta, ubicada en el cantón Colta de la provincia de Chimborazo. Esta laguna es un ecosistema lentico dulceacuícola que se mantiene inundada todo el año; tiene 2800 metros de largo por 1000 metros de ancho y está rodeada de varias colinas, donde habitan varias comunidades indígenas (GAD Colta, 2016; Guaminga, 2019) (ver Figura 1).

\section{Muestras}

Para realizar la investigación se realizaron cuatro campañas de muestreos entre los años 2019 y 2021 en la laguna Colta, en diferentes épocas del año, a fin de poder incluir muestreos en la época de lluvia y muestreos durante la época de verano. Las muestras de agua se recolectaron por duplicado en 5 sitios seleccionados a lo largo y ancho de la laguna a nivel de la superficie. Se identificaron las coordenadas geográficas de los sitios de recolección mediante un GPS marca Hanna (ver Tabla 1).

En cada ocasión se recolectaron muestras de agua de 1 litro en cada uno de los sitios de muestreo seleccionados. Para la recolección de las muestras se utilizaron frascos esterilizados y un muestreador de agua Van Dorn. Las muestras se etiquetaron con sus respectivas condiciones, trasladándose bajo refrigeración en una cava hasta el laboratorio, donde se realizaron los análisis microbiológicos dentro de las 24 horas luego de la toma (NTE INEN 2169, 2013; NTE INEN 2176, 2013).

\section{METODOLOGÍA}

\section{Análisis fisicoquímico in situ del agua de la laguna Colta, provincia de Chimborazo, Ecuador}

Los análisis fisicoquímicos se realizaron in situ en el agua de la laguna Colta, en cada uno de los puntos de muestreos seleccionados (tabla 1), utilizando para ello 


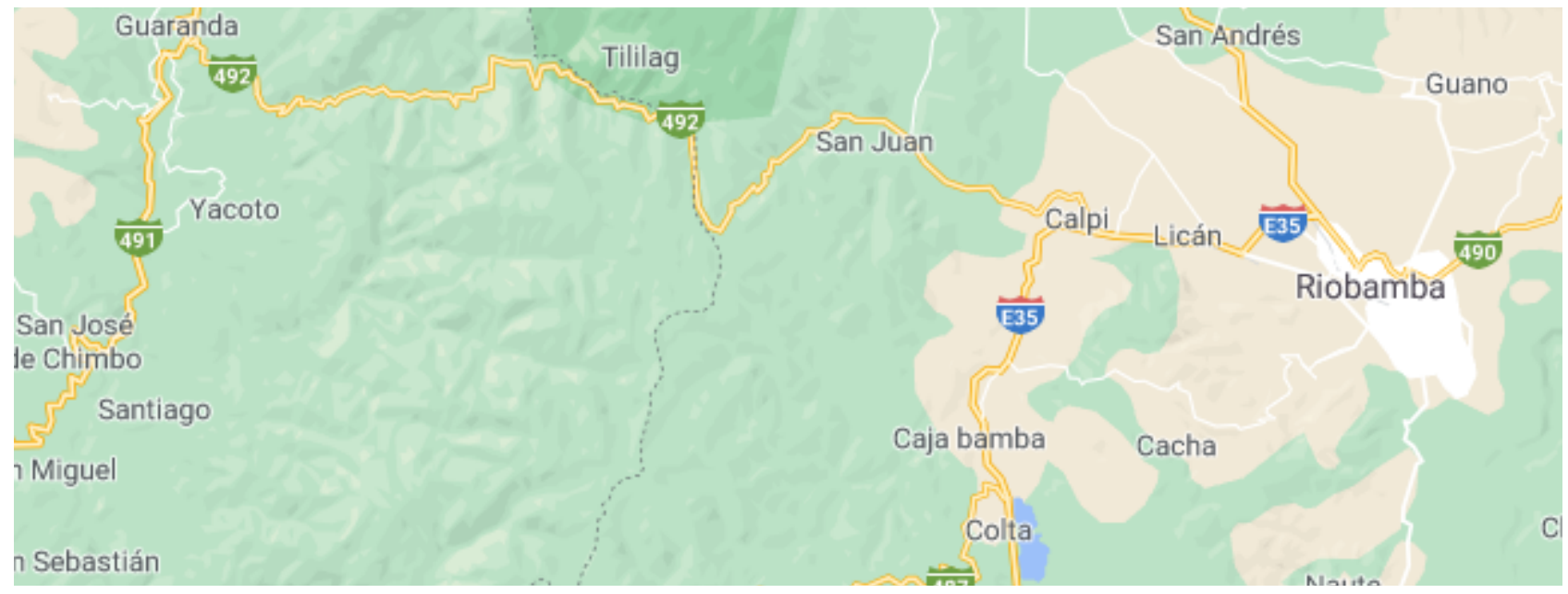

Figura 1. Ubicación geográfica de la laguna Colta. Provincia de Chimborazo, Ecuador (Google Maps, 2021).

Tabla 1. Geolocalización de los puntos de muestreos en la laguna Colta (Chimborazo, Ecuador)

\begin{tabular}{llll}
\hline $\begin{array}{l}\text { Puntos de muestreo } \\
\text { (Laguna de Colta) }\end{array}$ & $\begin{array}{l}\text { Coordenadas } \\
\mathbf{X}(\mathbf{m})\end{array}$ & $\begin{array}{l}\text { Coordenadas } \\
\mathbf{Y}(\mathbf{m})\end{array}$ & $\begin{array}{l}\text { altitud } \\
(\mathbf{m} \text { s. n. m.) }\end{array}$ \\
\hline P1 (norte de la laguna) & 749313,6 & 9808261,5 & 3310 \\
P2 (sur de la laguna) & 749467,1 & 9808127,3 & 3307 \\
P3 (este de la laguna) & 749565,3 & 9807537,5 & 3311 \\
P4 (oeste de la laguna) & 749243,1 & 9808418,1 & 3309 \\
P5 (centro de la laguna) & 749719,4 & 9808849,8 & 3312 \\
\hline
\end{tabular}

Tabla 2. Valores promedios totales de los resultados de los parámetros fisicoquímicos evaluados en la laguna Colta (Chimborazo, Ecuador)

\begin{tabular}{llllll}
\hline $\begin{array}{l}\text { Puntos de } \\
\text { muestreo }\end{array}$ & pH promedio & $\begin{array}{l}\text { Temperatura pro- } \\
\text { medio }\left({ }^{\circ} \mathbf{C}\right)\end{array}$ & $\begin{array}{l}\text { Conductividad eléc- } \\
\text { trica promedio } \\
(\boldsymbol{\mu s} / \mathbf{c m})\end{array}$ & $\begin{array}{l}\text { Sólidos totales } \\
\text { disueltos promedio } \\
(\mathbf{m g} / \mathbf{L})\end{array}$ & $\begin{array}{l}\text { Transparencia } \\
\text { sEECCHI pro- } \\
\text { medio }(\mathbf{m})\end{array}$ \\
\hline 1 & $8,94 \pm 0,17$ & $18,11 \pm 0,28$ & $10,79 \pm 70,8$ & $718 \pm 71,2$ & $0,94 \pm 0,14$ \\
2 & $9,1 \pm 0,13$ & $18,31 \pm 0,38$ & $1095 \pm 97,07$ & $742 \pm 93,65$ & $1,17 \pm 0,22$ \\
3 & $8,72 \pm 0,11$ & $17,78 \pm 0,33$ & $1104 \pm 13,97$ & $635 \pm 54,89$ & $1,31 \pm 0,13$ \\
4 & $8,69 \pm 0,13$ & $18,01 \pm 0,23$ & $1123 \pm 139,72$ & $680 \pm 107,1$ & $1,43 \pm 0,17$ \\
5 & $8,91 \pm 0,17$ & $17,36 \pm 0,58$ & $1024 \pm 85,49$ & $627 \pm 51,42$ & $2,85 \pm 0,47$ \\
Promedio & $8,87 \pm 0,17$ & $17,91 \pm 0,36$ & $1085 \pm 3762$ & $680 \pm 50,3$ & $1,54 \pm 0,75$ \\
\hline
\end{tabular}

un equipo medidor multiparámetro de marca HANNA. Las lecturas de cada uno de los parámetros investigados: $\mathrm{pH}$, temperatura, conductividad eléctrica, sólidos totales disuelto y transparencia se tomaron por duplicado (Bedoya, 2015). En el caso de la transparencia se utilizó el disco Secchi de acuerdo con lo indicado por Bedoya (2015).

\section{RESULTADOS Y DISCUSIÓN}

Análisis fisicoquímico del agua de la laguna Colta, provincia de Chimborazo, Ecuador

En la tabla 2 se muestran los resultados promedios obtenidos en cada punto de muestreo durante las cuatro campañas de recolección de muestras realizadas entre los años 2019 y 2021 (año 2019, 2 muestreos; 
año 2020, 1 muestreo; año 2021, 1 muestreo) en la laguna Colta, respecto a los parámetros fisicoquímicos analizados (ver Tabla 2).

$\mathrm{El} \mathrm{pH}$ fue uno de los factores fisicoquímicos evaluados en el agua de la laguna Colta. Se obtuvo un valor promedio global para el agua de la laguna de $8,87, \mathrm{pH}$ considerado alcalino y que se encuentran dentro de los límites máximos permisibles según la normativa ambiental ecuatoriana vigente, que establece valores entre 6,50-9,00 para cuerpos de agua utilizados con fines recreativos (Tulsma, 2005).

Varios autores en diversas partes del mundo han indicado que las aguas de este tipo de ecosistemas lacustres presentan valores de $\mathrm{pH}$ entre 6 y 9 , valores similares a los encontrados en el presente estudio (Quirós et al., 2002; Locascio de Mitrovich, 2005; Roldan y Ramírez, 2008; Sierra, 2011; Toro et al., 2012; Minor et al., 2019; Ohba et al., 2020; Verma and Prakash, 2020; Saber et al., 2020; González et al., 2020).

Una posible explicación para los valores $\mathrm{pH}$ alcalino observados pudiera estar en el hecho de que una alta actividad fotosintética, debida a la comunidad de algas y de bacterias que se encuentran en la laguna, pudiera sobresaturar de oxígeno al sistema acuático, provocando agotamiento de dióxido de carbono y con ello un aumento de los valores del pH del agua (Roldán y Ramírez, 2008).

La temperatura fue otro de los parámetros fisicoquímicos analizados, observándose un valor promedio global para la laguna de $17,91^{\circ} \mathrm{C}$, lo cual equivale a un agua con una temperatura templada. Los valores de temperatura obtenidos a lo largo de la investigación indican un patrón térmico estable con pocas fluctuaciones anuales (Tabla 2).

Se ha señalado que las cuencas de los lagos de alta montaña son relativamente pequeñas en comparación con los lagos de las zonas bajas y, como resultado, las características atmosféricas y climáticas de la zona tienen una gran influencia en las características de temperatura del agua embalsada (Roldán y Ramírez, 2008; Torres, 2016; Ventura, 2018; Rascón et al., 2021).

Los resultados obtenidos en esta investigación, relacionados con la temperatura del agua de la laguna Colta, son similares a los obtenidos por otros investigadores en lagos de alta montaña de Ecuador y de otras partes del mundo (Locascio de Mitrovich, 2005; Meneses, 2013; Beltrán, 2020; Ohba et al., 2020).

Dentro de los factores fisicoquímicos investigados estuvo también la conductividad eléctrica; los resultados obtenidos indican que un valor promedio para la conductividad eléctrica del agua de la laguna fue de $1085 \mu \mathrm{S} / \mathrm{cm}$ (Tabla 2).
Los valores encontrados en la conductividad eléctrica indican que se trata de un agua de mineralización alta (conductividad eléctrica superior a $1000 \mu \mathrm{S} /$ $\mathrm{cm})$ y no sería apta para consumo humano de acuerdo con la normativa nacional e internacional (Rodier, 1998; Roldán y Ramírez, 2008; Tulsma, 2015). Los resultados obtenidos son significativamente mayores a lo que se han indicado para diversos lagos de alta montaña en Ecuador y en otras partes del mundo, donde se observan valores entre 280 y $337 \mu S / \mathrm{cm}$ (Pawar, 2010; Toro et al., 2012; Ohba et al., 2020) y similares a los señalados en el lago Cuicocha en Ecuador (Gunkel y Beulker, 2009).

Los resultados obtenidos en la conductividad eléctrica, pudiera indicar la actividad de procesos geoquímico y geomicrobiológicos dentro de la estructura del lago y/o un problema de arrastre de sedimentos de las zonas aledañas (Rodríguez et al., 2010; Miot et al., 2016; Torres, 2016; Ohba et al., 2020).

Otro de los aspectos fisicoquímicos estudiados fue la cantidad de sólidos disueltos presentes en el agua de la laguna Colta, obteniéndose un valor promedio general para la laguna de $680 \mathrm{mg} / \mathrm{L}$ (tabla 2). Los resultados obtenidos son similares a los observados para el lago San Pablo (Gunkel, 2003) y mucho menores que los encontrados en Cuicocha (Gunkel y Beulker, 2009).

Los altos valores obtenidos de sólidos disueltos en el agua de la laguna Colta pueden deberse probablemente a la descarga de desechos domésticos y al arrastre de la escorrentía de abonos agrícolas utilizados por los habitantes de la zona, situación que pudiera estar evidenciando un problema significativo de aumento en los niveles de eutroficación en la laguna (Sierra, 2011).

En los análisis fisicoquímicos realizados se incluyó también la determinación del índice de transparencia del agua, utilizando el indicador Secchi que permite inferir el estado eutrófico de los ecosistemas acuáticos con base en su valor (Roldan y Ramírez, 2008). El valor promedio total obtenido en el agua de la laguna Colta para el índice Secchi fue de 1,54 metros (Tabla 2).

Se ha señalado que los valores en el índice Secchi menores a 1,5 metros, indicarían la presencia de un ecosistema lacustre hipertrófico, valores entre 1,5 a 3,0 metros un ecosistema eutrófico, entre 3 y 6 metros ecosistemas mesotróficos, de 6 a 12 metros ecosistemas oligotrofos y valores superiores a 12 metros ecosistemas ultra oligotróficos (Roldán y Ramírez, 2008; Bedoña, 2015; López y Madroñero, 2015). Los datos obtenidos apuntan a que estamos en presencia de un lago eutrófico con valores en el índice de Secchi entre 
1,5 y 3,0 metros. Existen diferencias en las consideraciones de eutroficación en la laguna Colta; algunos investigadores la clasifican como una laguna de aguas oligotróficas (Rodríguez, 2019).

La calidad de los cuerpos de agua dulce, como es el caso del agua de lagos y lagunas, se puede determinar analizando dos componentes básicos, las características fisicoquímicas y la población de microorganismos presentes (Sierra, 2011), razón por la cual en el trabajo se realizó primero un estudio fisicoquímico.

\section{CONCLUSIONES}

Los resultados obtenidos en los parámetros fisicoquímicos estudiados evidencian que la laguna Colta es un ecosistema acuático en un estado eutrófico con una alta productividad biológica y con características fisicoquímicas estables en las diferentes zonas de la laguna, a lo largo del año y de las estaciones climáticas que se presentan en la región. Los resultados obtenidos en las condiciones fisicoquímicas del agua de la laguna Colta permiten predecir la presencia en este ecosistema de una microbiota bacteriana aerobia mesófila escasa y poco diversa.

\section{AGRADECIMIENTO}

A la Escuela Superior Politécnica de Chimborazo por las facilidades otorgadas para la realización del presente trabajo.

\section{REFERENCIAS}

Bedoya. 2015. Estandarización de las metodologías para el análisis de $\mathrm{pH}$, alcalinidad y turbidez en muestras de agua potable en el laboratorio de la Asociación Municipal de Acueductos Comunitarios de Dosquebradas (AMAC) (Tesis doctoral). Universidad Tecnológica de Pereira. Facultad de Tecnologías. Tecnología Química. Pereira, Colombia.

Dar, S. A., Bhat, S. U., Aneaus, S., Irfan Rashid. 2020. A geospatial approach for limnological characterization of Nigeen Lake, Kashmir Himalaya. Environmental Monitoring Assessment, 192, 121. https://doi.org/10.1007/ s10661-020-8091-y

Gómez, G. 2018. Determinación del estado trófico actual de la laguna Cube a través de la cuantificación de parámetros químicos (fosfatos, nitratos, clorofila «a») y transparencia Secchi (Tesis de pregrado). Universidad Central del Ecuador. Quito, Ecuador.

González, M., Acuña, J., Escobar, J., Viteri, F., Villacís, L., Parra, Y., Araujo, L., Araque, J., Andueza, F. 2020. Calidad fisicoquímica del agua de la laguna volcánica cratérica Quilotoa. Revista Perspectiva, 21(1), 71-83. Cotopaxi. Ecuador.

Granados, I., Toro, M. 2000. Conservación de los lagos y humedales de alta montaña de la península Ibérica (pp. 33-50). Madrid, España: Servicio de Publicaciones, Universidad Autónoma de Madrid,
Gunkel, G. 2003. Limnología de un lago tropical de alta montaña en Ecuador: características de los sedimentos y tasa de sedimentación. Revista Biología Tropical, 51, 381-390.

Gunkel, G., Beulker, C. 2009. Limnology of the crater lake Cuicocha, Ecuador, a coldwater tropical lake. International Review of Hydrobiology, 94, 103-125.

INEN. 2013. NTE INEN 2169:2013. Agua. Calidad del agua. Muestreo. Manejo y conservación de muestras (1. ${ }^{\mathrm{a}}$ ed.). Instituto Ecuatoriano de Normalización. Norma 2169. Quito-Ecuador.

INEN. 2013. NTE INen 2176:2013. Agua. Calidad del Agua. Muestreo de técnicas de Muestreo (1. ${ }^{\mathrm{a}}$ ed.). Instituto Ecuatoriano de Normalización. Norma 2176. Quito-Ecuador.

Locascio de Mitrovich, C., Villagra de Gamundi, A., Juárez, J., Ceraolo, M. 2005. Características limnológicas y zooplancton de cinco lagunas de la Puna-Argentina. Ecología en Bolivia, 40(1), 10-24.

López-Martínez M. L., Madroñero Palacios S. M. 2015. Estado trófico de un lago tropical de alta montaña: caso laguna de La Cocha. Ciencia e Ingeniería Neogranadina, 25(2), 21-42. http://dx.doi.org/10.18359/rcin.1430

López-Martínez, M. L., Jurado-Rosero, G. A., Páez-Montero, I. D. y Madroñero-Palacios, S. M. 2017. Estructura térmica del lago Guamués, un lago tropical de alta montaña. Luna Azul, 44, 94-119. https://doi.org/10.17151/luaz.2017.44.7.

Meneses, L. 2013. Bacterioplancton de tres lagunas de alta montaña tropical andina en el departamento de Boyacá, Colombia (Tesis doctoral). Universidad Pedagógica y Tecnológica de Colombia. Tunja, Colombia.

Minor, E. C., Tennant, C. J. y Brown, E. T. 2019. A seasonal to interannual view of inorganic and organic carbon and $\mathrm{pH}$ in western Lake Superior. Journal of Geophysical Research: Biogeosciences, 124, 405-419. https://doi. org/10.1029/2018JG004664

Miot, J., Jézéquel, D., Benzerara, K., Cordier, L., Rivas-Lamelo, S., Skouri-Panet, F., Férard, C., Poinsot, M., Duprat, E. 2016. Mineralogical diversity in lake Pavin: Connections with Water Column Chemistry and Biomineralization. Processes Minerals, 6, 24-43. https://doi.org/10.3390/ $\min 6020024$

Ohba, T., Ooki, S., Oginuma, Y., Yoshida, H., Ntchantcho, R., Ako, A., Tawedi, R., Ayissi, M., Anyileke, G., Hell, J. 2020. Temperature and electrical conductivity of water in lake Nyos transmitted by an automatic observation buoy. Journal of African Earth Sciences, 172, 1-8.

Pawar, A. L. 2010. Seasonal variation in physicochemical quality of Lonar lake water. Journal of chemical and pharmaceutical research, 2(4), 225-231.

Quirós, R., Rosso, J. J., Rennella, A., Sosnovsky, A., Boveri, M. 2002. Análisis del estado trófico de las lagunas pampeanas. Interciencia, 27(11), 584-591. Argentina

Quirós, R. 2005. La ecología de las lagunas de las Pampas. Investigación y Ciencia. 1(6), 1-13.

Rascón, J., Corroto, F., Leiva-Tafur, D., Gamarra, O. 2021. Variaciones limnológicas espaciotemporales de un lago altoandino tropical al norte de Perú. Ecología Austral, 31, 343-356. 
Rodier, J. 1998. Análisis de las aguas naturales, residuales y aguas de mar (3. ${ }^{\text {e ed. }) . ~ B a r c e l o n a: ~ O m e g a ~ s . ~ A . ~}$

Roldán, G. y Ramírez, J. J. 2008. Fundamentos de limnología

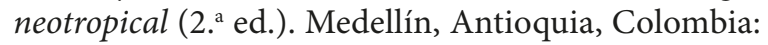
Universidad de Antioquia.

Rodríguez, S. 2019. Determinación del estado trófico actual de la laguna de Colta mediante la cuantificación de parámetros químicos (fosfatos, nitratos) y transparencia Secchi (Tesis de pregrado). Universidad Central del Ecuador. Quito, Ecuador.

Rodríguez, I., Duque, C., Calvache, C., López-Chicano, M. 2010. Causas de las variaciones de la conductividad eléctrica del agua subterránea en el acuífero Motril-Salobreña, Geogaceta, 49, 107-110.

Saber, A., James, D., Hannoun, I. 2020. Effects of lake water level fluctuation due to drought and extreme winter precipitation on mixing and water quality of an alpine lake. Case study: Lake Arrowhead, California. Science of the Total Environment, 714, 136-762. https://doi. org/10.1016/j.scitotenv.2020.136762.

Sierra, C. A. 2011. Calidad del agua: evaluación y diagnóstico (1. ${ }^{\text {a }}$ ed.). Medellín: Universidad de Medellín.

Tito-Ontaneda, B. M. 2018. Estudio morfométrico de la laguna Cube (Trabajo de titulación de pregrado). Carrera de Ingeniería Ambiental. Universidad Central del
Ecuador. Quito, Ecuador.

Toro, D., Salazar, M., Ocampo, D., Correa, R. y Salgado, P. 2012. Estudio limnológico de la laguna Negra, zona de amortiguación del PNN Los Nevados. Boletín Científico Museo de Historia natural, 2(16), 23-28.

Torres, J. 2016. Evaluación de impacto ambiental y plan de manejo ambiental del proyecto «Malecón escénico laguna de Colta», provincia de Chimborazo (Tesis de pregrado). Escuela Superior Politécnica de Chimborazo. Riobamba, Ecuador.

Tulsma. 2015. Anexo 1 del libro vi del Texto unificado de legislación secundaria del Ministerio del Ambiente. En Norma de calidad ambiental y de descarga de efluentes al recurso agua. Registro Oficial, p. 185. Quito, Ecuador.

Ventura, M. 2018. Los lagos de alta montaña como sensores del cambio climático. Impactos en los ecosistemas acuáticos. Centro de Estudios Avanzados de Blanes, csic, España. Disponible en: http://www.divulgameteo.es/ fotos/lecturas/Lagos-alta-montaña-CC.pdf.

Verma, A. K., Prakash, S. 2020. Limnological studies of Semara Taal. A wetland of district Siddharthnagar (U.P.), India. Journal of Fisheries and Life Sciences, 5(1), 15-19. 